\title{
Structure and charge monitoring of battery materials: long range vs. short range in Prussian Blue analogues
}

\author{
Marco Giorgetti \\ University of Bologna, Bologna, Italy; \\ marco.giorgetti@unibo.it
}

Nowadays, electrochemical energy storage plays a major societal role due to its widespread technological applications. Host nanostructured materials have a crystal structure with insertion sites, channels and/or interlayer spacings allowing the rapid insertion and extraction of lithium ions with generally little lattice strain. Therefore they are used as electrode materials for batteries. Dynamic processes occurring in batteries can be studied by operando modality. Operando experiments provide a realistic representation of the reaction behavior occurring at electrodes, which permits to checking the structural and electronic reversibility of a battery system, while at least one full cycle is performed. For all these reasons, ex situ studies, which reflect a given state of charge (SOC) of electrode materials are now complemented by operando measurements using complementary tools such as X-ray diffraction (XRD) and spectroscopic techniques such as X-ray absorption spectroscopy (XAS).

$\mathrm{X}$-ray absorption spectroscopy is a synchrotron radiation based technique that is able to provide information on local structure and electronic properties in a chemically selective manner. Operando synchrotron radiation x-ray powder diffraction (SR-XRPD) experiments allow monitoring the extended structure of a material during the intercalation/release process of ions.

The potentiality of the joint XAS-XRD approach in the newly proposed Prussian Blue-like cathodes materials for rechargeable batteries is here underlined.

Prussian blue analogous (PBAs) or metal hexacyanoferrates are bimetallic cyanides with a three-dimensional cubic lattice of repeating -Fe-CN-M-NC- units (where $\mathrm{M}=$ transition metals). Because of their peculiar structure exhibiting large ionic channels, interstices in the lattice and redox-active sites they have been proposed as active materials for electrodes in batteries. In our group, a series of PBAs have been synthesized, such as copper hexacyanoferrate (CuHCF), manganese hexacyanoferrate (MnHCF), titanium hexacyanoferrate (TiHCF), multi-metal doped hexacyanoferrate, as well as copper nitroprusside etc. In particular, this talk will be summarize results obtained in the case of copper hexacyanoferrate and copper nitroprusside, as well as the manganese hexacyanoferrate [1-5]. Sodiumrich manganese hexacyanoferrate $(\mathrm{MnHCF})$ is gaining consideration as battery materials for the versatility toward several chemistries beyond lithium, the ease of synthesis, as well as their affordable cost of production. MnHCF is constituted only by earth-abundant elements, and it displays high operational voltages and high specific capacities. Since PBAs act as sponge-like materials towards water molecules, also in case of short time exposure to contamination, and both the electrochemical behavior and the reaction dynamics are affected by interstitial/structural water and adsorbed water, the effect of hydration is critical in determining the electrochemical performance. The electrochemical activity of MnHCF without extensive dehydration was investigated by varying the interstitial ion content through a joint approach using operando x-ray absorption fine structure (XAFS) spectroscopy conducted at the XAFS beamline in ELETTRA and multivariate curve resolution with alternating least squares algorithm (MCR-ALS), with the intent to assess the structural and electronic modifications occurring during sodium release and lithium insertion as well as the overall dynamic evolution of the system. The study is also complemented to the and operando XRPD. It was found that only a minor volume change (about 2\%) is recorded upon cycling the electrode material against lithium.

[1] A. Mullaliu, G. Aquilanti, P. Conti, J. R. Plaisier, M. Fehse, L. Stievano, M. Giorgetti. Copper Electroactivity in Prussian Blue-Based Cathode Disclosed by Operando XAS. J. Phys. Chem. C, 122 (2018) 15868-15877.

[2] A. Mullaliu, M. Gaboardi, J. Rikkert Plaisier, S. Passerini, M. Giorgetti. Lattice Compensation to Jahn-Teller Distortion in Na-rich Manganese Hexacyanoferrate for Li-ion Storage: An Operando Study. ACS Appl. Energy Mater., 3, (2020) 5728-5733.

[3] A. Mullaliu, J. Asenbauer, G. Aquilanti, S. Passerini, M. Giorgetti. Highlighting the Reversible Manganese Electroactivity in Na-Rich Manganese Hexacyanoferrate Material for Li- and Na-Ion Storage. Small Methods, (2020) 1900529.

[4] M. Li, A. Mullaliu, S. Passerini, M. Giorgetti. Titanium Activation in Prussian Blue Based Electrodes for Na-ion Batteries: A Synthesis and Electrochemical Study. Batteries, 7 (2021) 5.

[5] A. Mullaliu, M.T. Sougrati, N. Louvain, G. Aquilanti, M.L. Doublet, L.Stievano, M. Giorgetti. The electrochemical activity of the nitrosyl ligand in copper nitroprusside: a new possible redox mechanism for lithium battery electrode materials? Electrochimica Acta, 257 (2017) 364-371.

The following researchers are kindly acknowledged: Angelo Mullaliu, Giuliana Aquilanti, Jasper R. Plaisier, Min Li, Stefano Passerini.

Keywords: Operando, XAS, XRPD, PBA, Batterie

Acta Cryst. (2021), A77, C350 